\title{
EVALUACIÓN DE LA TASA DE DEFECACIÓN Y DEL USO DE LETRINAS EN LA GUAGUA LOBA (Dinomys branickii RODENTIA: DINOMYIDAE)
}

\section{ASSESSMENT OF THE DEFECATION RATE AND THE LATRINE USE IN THE PACARANA (Dinomys branickii RODENTIA: DINOMYIDAE)}

\begin{abstract}
Karin Osbahr ${ }^{1}$
${ }^{1}$ Líder Grupo de Investigación en Fauna Silvestre. Universidad de Ciencias Aplicadas y Ambientales U.D.C.A. Dirección para correspondencia: Calle 222 No.55-37 Bogotá - D.C kosbahr@udca.edu.co
\end{abstract}

Rev. U.D.C.A Act. E Div. Cient. 13 (1): 57-66, 2010

\section{RESUMEN}

El conteo sistemático de grupos de excrementos ha sido utilizado para estimar parámetros poblacionales de diversas especies. La guagua loba (Dinomys branickii), se caracteriza por defecar en letrinas, que son empleadas repetitivamente por un grupo familiar, por lo que el muestreo de la materia fecal constituye una alternativa, más aún, cuando las poblaciones de la especie son pequeñas y con distribución discontinua; sin embargo, la técnica requiere del conocimiento de la tasa de defecación y de la morfología de la materia fecal desconocidas para la especie. El objetivo principal de este trabajo fue el de monitorear las letrinas de tres poblaciones con diferente número de individuos, entre machos y hembras de $D$. branickii, mantenidas en un ambiente seminatural, con el fin de generar información de la morfología y de la producción diaria de materia fecal. Para cada grupo de animales, se recolectó, se contó y se midió el total de cagarrutas depositadas por letrina. El número total de cagarrutas/animal/día, se obtuvo sirviendose de marcadores fecales. El promedio de las cagarrutas por letrina varían de acuerdo al tamaño del grupo. Las tasas de defecación individual mostraron diferencias estadísticamente significativas entre sexos. La forma cilíndrica de las cagarrutas de $D$. branickii muestra una clara diferencia entre la longitud y el ancho promedio. Las dimensiones de las cagarrutas variaron con mayor frecuencia en las letrinas que en las muestras individuales. La estimación del tamaño poblacional de D. branickii, a partir del uso de letrinas, es prometedora, pero requiere ser validada en su hábitat natural.

Palabras clave: Letrinas, tasa de defecación, guagua loba, Dinomys branickii.

\section{SUMMARY}

The systematic fecal pellet-group count has been used to estimate population parameters of various species. The Pacarana (Dinomys branickii) is characterized by defecating in latrines which are used repeatedly by a family group, so that sampling of the fecal pellets is an alternative, since the species lives in small populations with discontinuous distribution. However, the technique requires knowledge of the defecation rate and fecal morphology unknown for this species. The main objective of this study was to monitor the latrines in three populations of $D$. branickii with different numbers of individuals between males and females kept in a seminatural environment, in order to generate information on the morphology and the daily production of feces. For each group of animals the total number of droppings deposited at each latrine was collected, counted and measured. The total number of droppings/ animal/day was obtained using fecal markers. The mean droppings per latrine varied according to group size. Individual defecation rates showed statistically 
significant differences between sexes. The cylindrical shape of the droppings of $D$. branickii shows a clear difference between the average length and width. The dimensions of the droppings varied more frequently in the latrines than in the individual samples. The estimation of the population size of $D$. branickii based on the use of latrines is promising, but needs to be validated in its natural habitat.

Key word: Latrines, defecation rate, pacarana, Dinomys branickii

\section{INTRODUCCIÓN}

Para conocer la abundancia y la densidad local de las poblaciones en relación al área de distribución y al grado de especialización ecológica de una especie dada, han sido reportados diferentes métodos, tales como los conteos directos o las técnicas de captura, de marcaje y de recaptura, que posibiliten obtener datos precisos sobre el estado de las poblaciones de animales (Krebs, 1999). Cualquiera que sea el método empleado es claro que se requiere de información biológica básica de la especie en especial si se pretende obtener modelos que permitan realizar proyecciones a largo plazo de la dinámica poblacional y sobre la capacidad que tiene la especie para sobrevivir ante adversidades ambientales (Vucetich et al. 2000; Lindenmayer et al. 2003; McCarthy et al. 2003).

Dado que se trata de métodos costosos que implican el uso de tecnología avanzada, han tomado importancia las técnicas indirectas, entre las que se destaca el conteo de grupos de excrementos. Esta metodología facilita definir el número actual o estimado de determinada especie en un área dada, ofreciendo una medida objetiva de las variaciones y las fluctuaciones sustanciales en una población, a la vez, que ofrece datos sobre preferencias de hábitat y su utilización diferencial de acuerdo con patrones estacionales (Bennet et al. 1940; Riney, 1957). Aún cuando la técnica presenta algunas dificultades, como la pérdida de muestras, debida al lavado por lluvias (Barnes E Dunn, 2002) y al ataque por insectos coprófagos, el método presenta una ventaja considerable al tratarse de evidencias inertes, que pueden ser muestreadas, sistemáticamente, en el campo (Neff, 1968; Putman, 1984), ofreciendo un método robusto para el establecimiento del número de individuos en poblaciones con densidades bajas (Murray et al. 2002). El conteo de materia fecal, el tamaño de grupos de excrementos, la tasa de defecación y el uso de letrinas han sido utilizados para estimar parámetros poblacionales relacionados, incluso, con el uso del hábitat de diversas especies (Vernes, 1999; De Boer et al. 2000; Theuerkauf \& Ellenberg, 2000; Krebs et al. 2001; Lombardi et al. 2003; Homyack et al. 2006).

Algunos vertebrados se caracterizan por defecar en letrinas, visitadas repetitivamente por individuos de la misma especie. Muchos mamíferos emplean esta forma de defecación, para marcar los límites de su territorio. El conocimiento de las letrinas ofrece información sobre el número de animales que las han visitado y, por lo tanto, es posible extrapolar el tamaño de una población, a la vez, que permite determinar el uso del hábitat y evaluar la ecología y el comportamiento de una especie (Mykytowycz \& Gambale, 1969; Palomares, 1993; Roper et al. 1993; Irwin et al. 2004; Sprent et al. 2006).

La presencia de excrementos de una especie en un área dada está estrechamente ligada al comportamiento y a las características morfológicas y fisiológicas del sistema digestivo. Por lo tanto, el conteo de excrementos, como herramienta para establecer el tamaño y la distribución de una población, requiere del conocimiento preciso de la morfología y de las dimensiones de la materia fecal, de la especie de interés. En la medida en que se conocen igualmente las tasas de defecación y de descomposición de la materia fecal, se puede definir la metodología más apropiada para detectar la presencia de la especie a partir de la evidencia de defecación. En este sentido, el estudio de la materia fecal de animales mantenidos en cautiverio, en condiciones seminaturales, debe ser aprovechado, más aún, cuando no existen datos para las poblaciones silvestres (Chapman, 2004). Incluso, para establecer la presencia o ausencia de sesgos en la estimación del tamaño poblacional a partir de las heces, la técnica ha sido probada muestreando poblaciones de tamaño conocido en cautiverio (PérezMejía et al. 2004) o comparando los resultados con las estimaciones obtenidas con otras técnicas, indicando elevados márgenes de confiabilidad (Tuyttens et al. 2001; Cattadori et al. 2003; Scott Mills et al. 2005).

La guagua loba o pacarana (Dynomis branikii) es un roedor propio de la región andina, desde Venezuela hasta el piedemonte brasileño (White $E$ Alberico, 1992). Aún cuando el estimativo del tamaño de las poblaciones es 
esencial para la definición de medidas de conservación en especies amenazadas de extinción (Hochachka et al. 2000; Sulkava et al. 2008), esta información es escasa o inexistente para $D$. branickii, considerada en la literatura como una especie rara, con posibles densidades poblacionales bajas con tendencia a disminuir, por lo cual, es reportada en la lista roja global (IUCN, 2009) y colombiana (Alberico et al. 2006), de mamíferos amenazados. Estas aparentes poblaciones pequeñas, cuya distribución en Colombia parece ser discontinua, requieren de medidas urgentes para obtener información sobre el estado de conservación real de la especie. Siendo una especie nocturna, que habita en zonas montañosas con bosques subandinos y andinos densos y de difícil acceso, los métodos directos para la estimación del tamaño de las poblaciones son inapropiados (Chame, 2003). Sin embargo, el hecho que la especie utiliza letrinas que son visitadas repetitivamente por varios individuos que componen un grupo familiar (Osbahr E Bautista, 1998; Osbahr, 1999; Osbahr \& Restrepo, 2002), constituye una alternativa para el muestreo de la materia fecal. Por tal razón, el objetivo principal de éste trabajo fue el de estimar las tasas de defecación, monitoreando las letrinas de tres poblaciones de sexos diferentes de $D$. branickii, mantenidas en un ambiente seminatural, con el fin de generar información de la morfología y de la producción diaria de materia fecal, que permita adelantar futuros estudios, utilizando el conteo de cagarrutas por letrina, en condiciones naturales.

\section{MATERIALES Y MÉTODOS}

El estudio se realizó en un encierro de $250 \mathrm{~m}^{2}$, ubicado en la Unidad de Investigación en Fauna Silvestre de la Universidad de Ciencias Aplicadas y Ambientales U.D.C.A, donde se mantienen las poblaciones muestreadas de D. branickii en Bogotá. El encierro construido con muros laterales de ladrillo y de malla, está ambientado con suelo en tierra y pasto kikuyo (Kikuyuochloa clandestina), cuevas, troncos, bebederos y plantas, que ofrecen sombrío y refugio natural. Durante el estudio, se disminuyó gradualmente de 13 a cinco el número de ejemplares, para monitorear en un mismo encierro tres grupos de animales de sexos diferentes. De acuerdo con lo sugerido en estudios similares por Simonetti (1989) y Chapman (2004), se ofreció diariamente una dieta compuesta por habichuela (Phaseolus vulgaris), zanahoria (Daucus carota), yuca (Manhiot sp.), papa
(Solanum tuberosum), maíz en mazorca (Zea mays), banano (Musa paradisiaca), guatilla (Sechium edule), ahuyama (Cucurbita maxima), calabaza (Cucurbita pepo), lechuga (Lactuca sativa) y hoja de col (Brassica oleracea), la cual, cumple con los requerimientos en fibra establecidos para la especie (Osbahr $E$ Restrepo, 2002).

Para obtener el número total de cagarrutas/animal/día, se colectaron muestras de animales de edad y de sexo conocidos utilizando durante 25 días marcadores fecales, suministrando vía oral $1 \mathrm{~cm}^{3} /$ día de colorante verde para alimentos a un macho y dos hembras, seleccionados al azar, durante dos períodos diferentes, para evitar que las muestras de materia fecal se mezclaran. Siguiendo la metodología descrita por Delahay et al. (2000), se realizó una prueba, suministrando vía oral una mezcla de miel con escarcha plástica. Para obtener muestras de animales de sexo desconocido, se ofrecieron ad libitum junto con la dieta habitual de $500 \mathrm{~g}$ de remolacha (Beta vulgaris) sin procesar. Diariamente, se recogió de la letrina la materia fecal, contando el número de cagarrutas, con el marcador fecal. A partir de la tasa de defecación por animal, se evaluó si este parámetro permite estimar el tamaño de la población. Para tal efecto, se recolectaron y contaron durante 21 días y para cada población de tamaño conocido el total de cagarrutas depositadas por letrina.

En una letrina, se pueden encontrar cagarrutas de diferente tamaño (Figura 1), por lo cual, se siguió la metodología sugerida por Komers \& Brotherton (1997), con el fin de establecer si el tamaño es una variable confiable para determinar si las cagarrutas proceden de animales diferentes. Para este efecto se midió, empleando un calibrador, el ancho y el largo del total de las cagarrutas frescas con marcador fecal y del $10 \%$ de las cagarrutas de las muestras totales por letrina. Los valores obtenidos para los excrementos de animales de sexo conocido, se utilizaron para compararlos con las excretas de animales desconocidos. De manera complementaria, se llevaron a cabo observaciones directas del comportamiento de la especie al defecar, registrando, a su vez, la ubicación de las letrinas en el encierro. Para el análisis de resultados, se aplicó estadística descriptiva y pruebas de múltiples rangos, para la comparación entre las muestras. Las dimensiones de las cagarrutas, se analizaron aplicando pruebas de t-Student multiples (Ury, 1976; Sokal E Rohlf, 2000). 


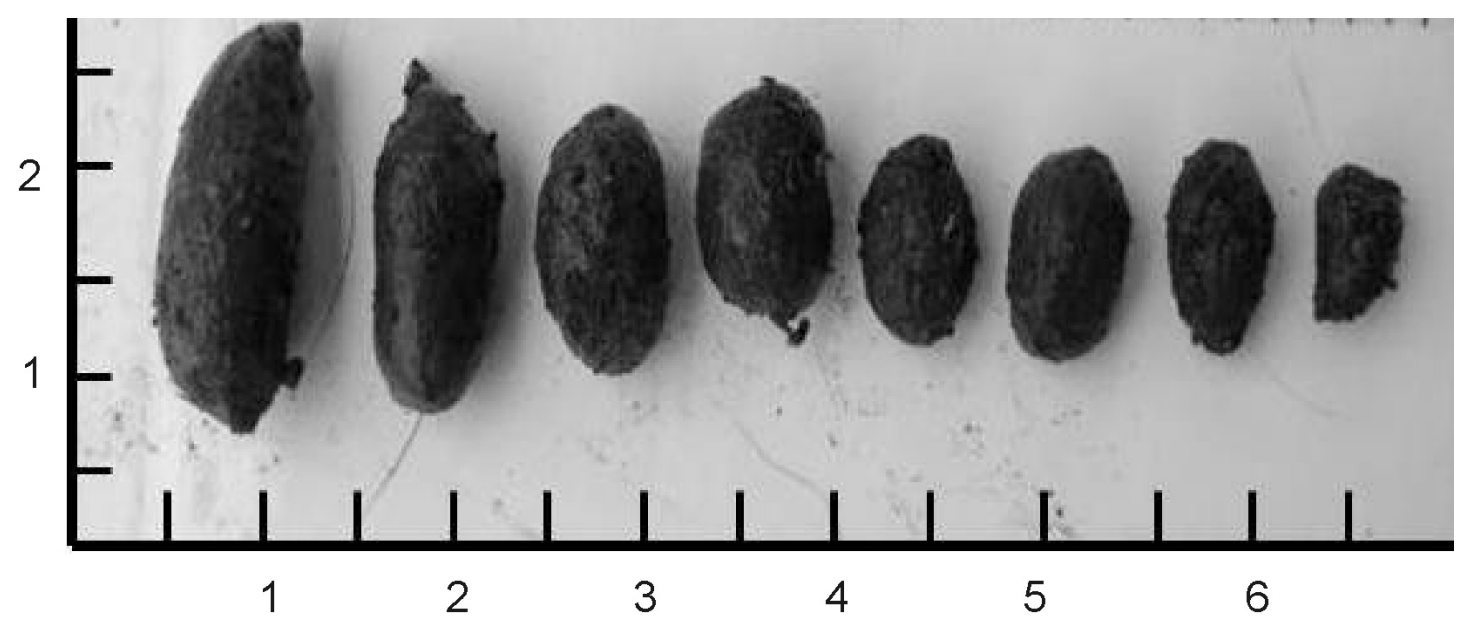

Figura 1. Variación en los tamaños de cagarrutas de $D$. branickii, en una letrina utilizada por varios individuos. La referencia de medida se encuentra expresada en $\mathrm{cm}$.

\section{RESULTADOS Y DISCUSIÓN}

El promedio y el rango del total de las cagarrutas por letrina varían de acuerdo al tamaño del grupo (Tabla 1). Teniendo en cuenta que la escasa información existente reporta que los grupos familiares de $D$. branickii están compuestos por dos a cinco individuos (Boher $\&$ Marin, 1988), aun cuando Woods (1984) menciona que se les puede ver solitarios o en parejas, se asumiría que la aplicación de la técnica del conteo de cagarrutas totales por letrina en condiciones de campo, arrojaría un estimativo apropiado del tamaño de los grupos, si se consideran los rangos y los valores promedio de cagarrutas por letrina, obtenidos para los grupos compuestos de siete y cinco individuos, respectivamente (Tabla 1). Teniendo en cuenta que el volumen defecado depende del tipo de alimentación ingerido y de su digestibilidad (Neff, 1968; Osbahr E Restrepo, 2002) es posible afirmar que aún cuando todos los grupos recibieron la misma dieta, la tasa individual de consumo dada por la eficiencia en el forrajeo (Stillman et al. 2000), modifica la producción diaria de materia fecal por letrina, utilizada por un grupo, lo cual, explicaría los coeficientes de variación obtenidos (Tabla 1). Esto, a su vez, se refleja al calcular en cada grupo la producción de cagarrutas/individuo/día, registrándose diferencias

Tabla 1. Estadísticos obtenidos para la tasa de defecación por cada grupo experimental. $\mathrm{N}=$ número de individuos; $\mathrm{D}=$ sexo desconocido.

\begin{tabular}{|c|c|c|c|c|c|c|c|c|c|}
\hline \multicolumn{4}{|c|}{ Cagarrutas/letrina/día } & \multicolumn{3}{|c|}{$\begin{array}{c}\text { Calculado } \\
\text { Cagarrutas/individuo/día }\end{array}$} & \multicolumn{3}{|c|}{$\begin{array}{l}\text { Muestreado } \\
\text { Cagarrutas/individuo/día }\end{array}$} \\
\hline $\mathrm{N}$ & 13 & 7 & 5 & 13 & 7 & 5 & 우 & 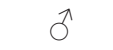 & D \\
\hline Promedio & 282,9 & 80,4 & 59,7 & 21,6 & 11,6 & 12,0 & 14,6 & 11,8 & 16,0 \\
\hline Desviación Estándar & 54,8 & 16,9 & 22,6 & 4,2 & 2,5 & 4,6 & 3,6 & 4,2 & 4,9 \\
\hline $\begin{array}{l}\text { Coeficiente de } \\
\text { Variación }\end{array}$ & $19,39 \%$ & $21,03 \%$ & $37,93 \%$ & $19,44 \%$ & $21,51 \%$ & $38,18 \%$ & $24,69 \%$ & $35,49 \%$ & $30,88 \%$ \\
\hline Mínimo & 130 & 57 & 32 & 10 & 8 & 6 & 7 & 5 & 7 \\
\hline Máximo & 395 & 118 & 98 & 30 & 17 & 20 & 25 & 19 & 25 \\
\hline \multicolumn{4}{|c|}{ Contraste } & $N=13-N=7$ & $N=13-N=5$ & $N=7-N=5$ & $q-0^{\lambda}$ & q - D & $\sigma^{\lambda}-\mathrm{D}$ \\
\hline \multicolumn{4}{|c|}{ LSD Fisher } & $10,04^{*}$ & $9,7^{*}$ & 0,4 & $2,72^{*}$ & 1,44 & $4,16^{*}$ \\
\hline \multicolumn{4}{|c|}{ ANOVA } & & & & \multicolumn{3}{|c|}{$F_{(2,72)}=6,05^{*}(p<0,05)$} \\
\hline
\end{tabular}


estadísticamente significativas entre los grupos de 13, siete y cinco individuos, con un promedio total calculado de 15,09 $\pm 6,0(n=63)$ (Tabla 1). De hecho, Murray et al. (2005) reportan diferencias significativas en la cantidad de cagarrutas/día, de acuerdo a la dieta ofrecida a Lepus americanus, en condiciones experimentales.

Los valores promedio $(14,1 \pm 4,6 n=75 ; C V=32,29 \%)$ obtenidos para la tasa de defecación individual, corroboran, igualmente, las variaciones en el volumen total defecado por letrina. Al comparar los valores muestreados frente a los calculados (Figura 2), se observa que las distribuciones de frecuencia son similares. Las tasas de defecación individual mostraron diferencias estadísticamente significativas entre sexos (Tabla 1). Estos resultados difieren de lo registrado por Murray op. cit. quienes en condiciones de una dieta conocida reportan similitudes en la producción diaria de cagarrutas por sexo; sin embargo, cabe anotar que a diferencia de $L$. americanus, $D$. branickii no es coprófago, por lo cual, no necesariamente se puedan comparar los dos modelos. Teniendo en cuenta que este estudio se realizó en condiciones de cautiverio es necesario recalcar que la estimación de la tasa de defecación obtenida para $D$. branickii no está exenta de sesgos, por lo que se debe considerar la variación de la tasa de defecación cuando se utilice esta técnica para hacer estimaciones del tamaño poblacional, en condiciones naturales.

Se logró verificar la bondad de $B$. vulgaris y del colorante para alimentos, como marcadores fecales, puesto que el color, bien sea rojo o verde, se presenta en las heces, aproximadamente, 36 horas después de la ingesta y se mantiene durante un período de dos a tres días. Es importante considerar que al emplear escarcha, se observó que este marcador se fija en la superficie exterior de la cagarruta, por lo que aumenta el riesgo de contaminar la letrina, dificultando la identificación individual. No obstante, Delahay et al. (2000) mencionan como ventaja, que la escarcha plástica ofrecida como marcador en cebos, no sería lavada por la lluvia, en caso de muestreos no continuos de las letrinas. En el evento específico del uso de $B$. vulgaris, como marcador, la técnica no garantiza que las cagarrutas de color rojo correspondan a un único animal, durante la totalidad de los días muestreados, pero la prueba de diferencia mínima significativa establece que no existen diferencias entre las cagarrutas/animal/día para las hembras y los datos obtenidos mediante este método,

Calculado

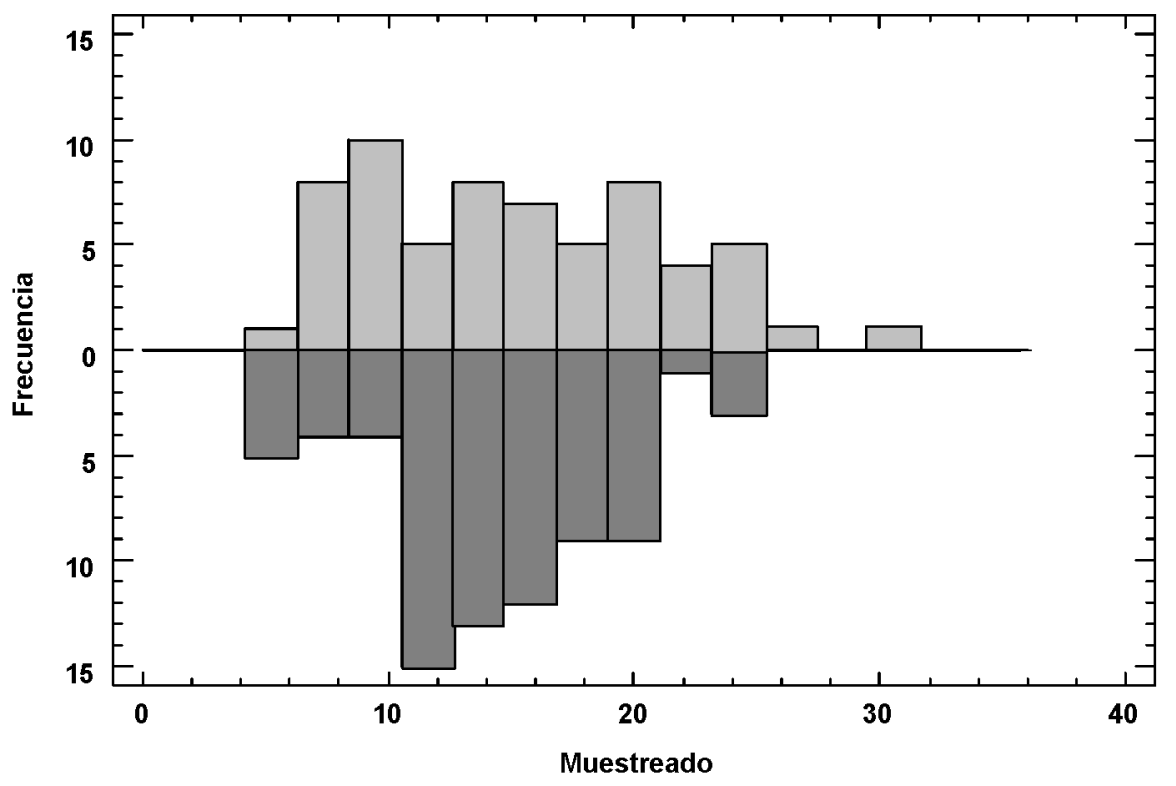

Figura 2. Distribución de frecuencia comparativa del número de cagarrutas /individuo/día muestreados en individuos de sexo conocido y calculados a partir del total de cagarrutas/letrina. 
para animales de sexo desconocido (Tabla 1). Dadas las diferencias halladas entre sexos, se podría asumir que dichas muestras provienen igualmente de una hembra. De acuerdo con Langbein et al. (1999), el conteo de cagarrutas marcadas puede ser de valor limitado al cuantificar la tasa de defecación y el uso de letrinas, ya que se registran variaciones significativas a corto plazo en el número de cagarrutas marcadas y cuantificadas en letrinas individuales. Los autores concluyen que estas variaciones pueden estar relacionadas con problemas prácticos en la aplicación de la técnica de conteo de cagarrutas marcadas que implican la dificultad de encontrar el total de excrementos durante los muestreos, lo cual, depende de la ubicación de la letrina y de la presencia o ausencia de lluvias. De tal manera que los marcadores fecales ofrecen datos confiables, pero se deben realizar las repeticiones necesarias en los conteos, para disminuir la varianza.

Las cagarrutas de $D$. branickii, se caracterizan por ser consistentes, de forma cilíndrica y bajas en humedad (Osbahr \& Bautista, 1998; Osbahr \& Restrepo, 2002), encontrándose en el grupo III de las categorías de morfología fecal, establecidas por Chame (2003). La forma cilíndrica de las cagarrutas de $D$. branickii muestra una clara diferencia entre la longitud y el ancho promedio $(23,71 \pm 5,90 \mathrm{CV}=24,9 \% ; 13,82 \pm 2,20$ $C V=15,9 \% n=905$, respectivamente). En la figura 3, se presentan las diferencias en los promedios al analizar por separado las muestras obtenidas en las letrinas y en los individuos muestreados con el marcador fecal.

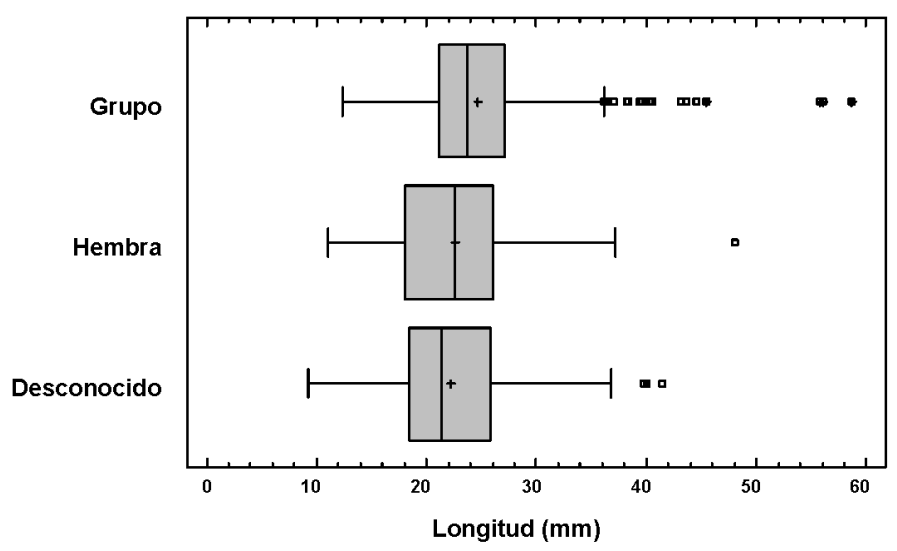

Es de resaltar que en las letrinas se encontraron mayor número de valores atípicos para la longitud, lo cual, nuevamente, puede estar relacionado con el tipo de alimento seleccionado por los diferentes individuos, durante el muestreo. Las dimensiones de las cagarrutas variaron con mayor frecuencia en las letrinas que en las muestras individuales, aún cuando para ambos casos se halló un elevado porcentaje de diferencias, estadísticamente significativas, en al menos una medida (Figura 4). Así mismo, el 24,4\% de las muestras entre individuos y el $18,1 \%$ dentro de la población mostraron variaciones estadísticamente significativas en las dos variables. Las diferencias interindividuales en el tamaño de las cagarrutas pueden ser detectadas por medio de la longitud y el ancho, ofreciendo una aproximación a la composición del grupo de $D$. branickii que frecuenta una letrina. De acuerdo con Coe E Carr (1983) y Zahratka $\mathcal{E}$ Buskirk (2007), estas diferencias en el tamaño de las cagarrutas pueden estar ligadas a la masa corporal de los individuos, lo cual, debe ser analizado, en detalle, para la guagua loba.

El monitoreo del comportamiento indica que los animales presentan ciclos individuales al defecar, es decir, que el uso de la letrina por parte de los individuos que conforman un grupo familiar, ocurre en tiempos diferentes, con una duración de 10 a 15 minutos por animal. Este comportamiento permite identificar, en una misma letrina, grupos de excrementos, los cuales, pertenecen a individuos distintos. Las observaciones muestran una clara predilección de $D$. branickii para

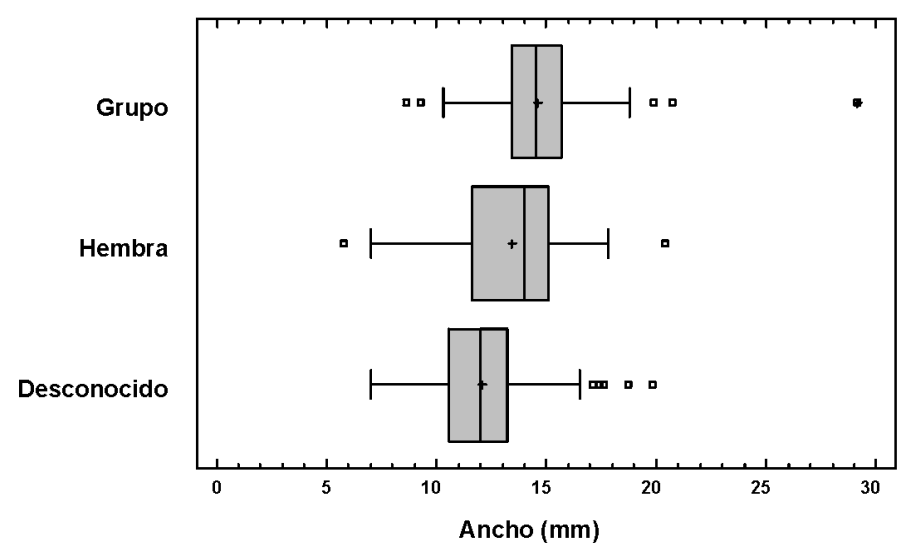

Figura 3. Comparación de los estadísticos descriptivos de la longitud y del ancho de las cagarrutas muestreadas en letrinas y en individuos de $D$. branickii. Promedio (+), media (-), desviación estándar (T), valores atípicos ( $\square$ ). Grupo $n=549$, Hembra $n=153$, Desconocido $n=203$. 


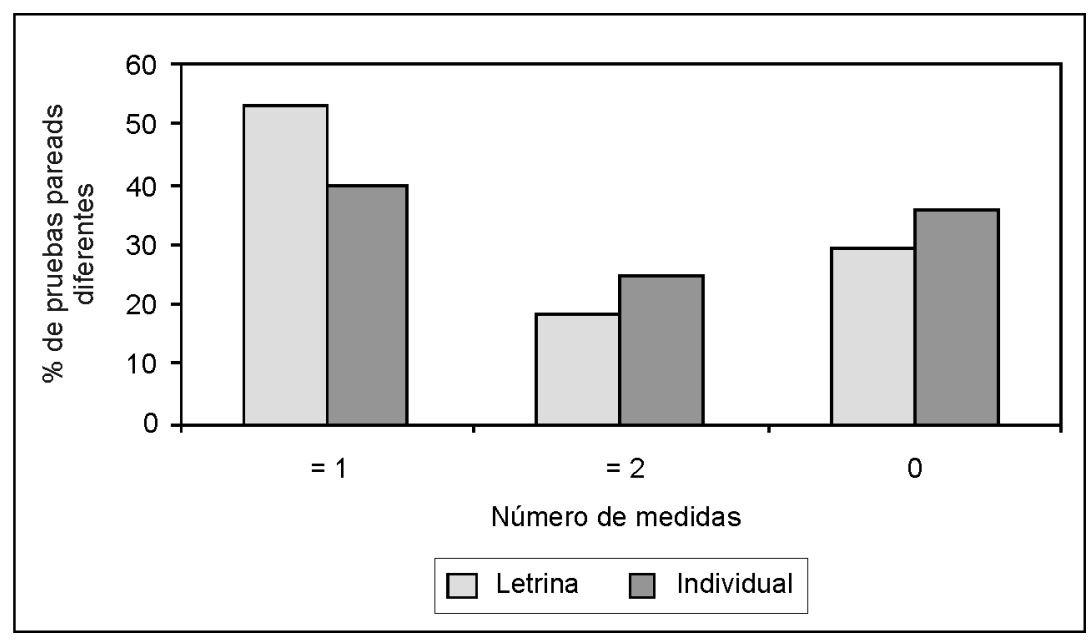

Figura 4. Porcentaje de las comparaciones pareadas, en las que se detectaron diferencias estadísticamente significativas en el tamaño de las cagarrutas de $D$. branickii. Se presentan las comparaciones, donde las cagarrutas difieren de manera significativa en, por lo menos, una, dos 0 ninguna de las medidas.

ubicar las letrinas en el interior de las cuevas, no utilizadas para el descanso, o de trepar para defecar a los troncos dispuestos, como enriquecedores ambientales. Reportes de la ubicación de las letrinas en refugios rocosos utilizados por otros roedores caviomorfos, tales como Cuniculus taczanowskii (Osbahr E Ortíz, 2007) y Kerodon rupestris (Araújo et al. 1993), indican que en condiciones naturales $D$. branickii igualmente busque sitios de difícil acceso, protegidos por troncos y rocas o elevada cobertura vegetal. La valoración realizada por Espirito-Santo et al. (2007) de las variables que inciden en general en la ubicación de las letrinas, revela que las zonas con baja presencia humana, difícil acceso y alta densidad en el sotobosque, influencian la selección de los lugares y refuerzan su uso.

Varios autores (Eisenberg E Kleiman, 1972; Johnson, 1973; Zollner et al. 1996) han sugerido que las letrinas deben ser entendidas como centros de intercambio de información, tal como ocurre en diversas especies de mamíferos, que conviven en colonias. Este comportamiento permite asumir que $D$. branickii puede estar definiendo su territorio grupal con este sistema, al ser utilizadas las letrinas por todos los integrantes de un grupo familiar, compuesto por machos y por hembras de diferentes edades, cumpliendo así una función para el intercambio de información olfativa. En el caso de los conejos europeos (Oryctolagus cuniculus) ha sido posible detectar que esta especie defeca en letrinas, marcadas con las glándulas anales (Sneddon, 1991).
D. branickii, probablemente, utiliza algún tipo diferente de marcaje de las letrinas, puesto que las disecciones anatómicas realizadas en individuos adultos (Osbahr et al. 2009) no muestran la presencia de glándulas anales. No obstante, durante este estudio fue posible observar que el grupo cambia con regularidad el lugar para defecar, con lo cual, se asume que el olor incita a los demás miembros a utilizar la nueva letrina. Cabe mencionar que las secreciones nasales asociadas a comportamientos agonales o exploratorios de la especie (López et al. 2000) no se producen al defecar, por lo que se descartaría que $D$. branickii utilice este tipo de secreción para marcar las heces. Observaciones adicionales no evaluadas en este estudio indican que las crías siguen a la hembra a la letrina, incluso, cuando ésta defeca en un nuevo lugar. Considerando que López et al. (2000) sugieren que las hembras podrían ser el eje de la estructura social en $D$. branickii, este comportamiento indicaría que son ellas las que definen la ubicación de la nueva letrina.

Los valores obtenidos durante el presente estudio ofrecen una guía para estimar el tamaño poblacional a partir de las letrinas. Aún cuando la descomposición de los cagarrutas de materia fecal puede jugar un papel importante en la desviación en la información sobre el tamaño en las poblaciones, en el caso de $D$. branickii favorece el hecho que las cagarrutas contienen un elevado porcentaje en fibra (Osbahr $\mathcal{E}$ Restrepo, 2002), ya que tienden a secarse y endurecerse; sin embargo, 
considerando la elevada humedad en las zonas en las que se distribuye la especie, se podría presentar una mayor acción por parte de organismos descomponedores, tales como hongos y bacterias. La estimación del tamaño poblacional de $D$. branickii, a partir del uso de letrinas, es prometedora, pero requiere ser validada en su hábitat natural para poder identificar prioridades de manejo, para esta especie vulnerable a la extinción.

Conflicto de intereses: El manuscrito fue revisado y preparado por el autor quien declara que no existe ningún conflicto de intereses que ponga en riesgo los resultados presentados. Financiación: Este estudio fue financiado por las instituciones que conforman el Pacarana Conservation Comité, con sede en la U.D.C.A.

\section{BIBLIOGRAFÍA}

\section{ALBERICO, M.; OSBAHR, K.; GONZÁLEZ-HERNÁN-} DEZ, A. 2006. Guagua loba (Dinomys branickii). En: Libro rojo de los mamíferos de Colombia. 1 ed. Bogotá, Panamericana Forma e Impresos S.A. p.294-297.

2. ARAÚJO, A.J.G.; RANGEL, A.; FERREIRA, L.F. 1993. Animal parasitic infection and climate change in Northeastern Brazil. Mem. Inst. Oswaldo Cruz. 88:577-579.

3. BARNES, R.F.W.; DUNN, A. 2002. Estimating forest elephant density in Sapo National Park (Liberia) with a rainfall model. Afr. J. Ecol. 40:159-163.

4. BENNET, L.J.; ENGLISH, P.F.; MCCAIN, B. 1940. A study of deer population by use of pellet-group counts. J. Wildlife Managm. 4(4):398-403.

5. BOHER, S.; MARIN, B. 1988. El pacarana (Dinomys branickii) en Venezuela. Natura. 84:14-18.

6. CATTADORI, I.M.; HAYDON, D.T.; THIRGOOD, S.J.; HUDSON, P.J. 2003. Are indirect measures of abundance a useful index of population density? The case of red grouse harvesting. Oikos. 100:439-446.

7. CHAME, M. 2003. Terrestrial mammal feces: A morphometric summary and description. Mem. Inst. Oswaldo Cruz, Rio de Janeiro. 98(Suppl.I):71-94.
8. CHAPMAN, N. 2004. Faecal pellets of Reeves' muntjac, Muntiacus reevesi: defecation rate, decomposition period, size and weight. Eur. J. Wildl. Res. 50:141-145.

9. COE, M.J.; CARR, R.D. 1983. The relationship between large ungulate body weight and faecal pellet weight. Afr. J. Ecol. 21(3):165-174.

10. DE BOER, W.F.; NTUMI, C.P.; CORREIA, A.U.; MAFUCA, J.M. 2000. Diet and distribution of elephant in the Maputo Elephant Reserve, Mozambique. Afr. J. Ecol. 38:188-201.

11. DELAHAY, R.J.; BROWN, I.A.; MALLINSON, P.J.; SPYVEE, P.D.; HANDOLL, D.; ROGERS, L.M.; CHEESEMAN, C.L. 2000. The use of marked bait in studies of the territorial organization of the European badger (Meles meles). Mammal Review. 30:73-87.

12. EISENBERG, J.F.; KLEIMAN, D.G. 1972. Olfactory communication in mammals. Ann. Rev. Ecol. and System. 3:1-32.

13. ESPIRITO-SANTO, C.; ROSALINO, L.M.; SANTOSREIS, M. 2007. Factors affecting the placement of common genet latrine sites in a Mediterranean landscape in Portugal. J. Mammalogy. 88(1):201207.

14, HOCHACHKA, W.M.; MARTIN, K.; DOYLE, F.; KREBS, C.J. 2000. Monitoring vertebrate population using observation data. Can. J. Zool. 78:527-529.

15. HOMYACK, J.A.; HARRISON, D.J.; LITVAITIS, J.A.; KROHN, W.B. 2006. Quantifying densities of snowshoe hares in Maine using pellet plots. Wildlife Soc. Bull. 34(1):74-80.

16. IRWIN, M.T.; SAMONDS, K.E.; RAHARISON, J.L.; WRIGHT, P. 2004. Lemur latrines: Observations of latrine behavior in wild primates and possible ecological significance. J. Mammalogy. 85(3):420427.

17. IUCN. 2009. IUCN Red List of Threatened Species. Version 2009.2. Disponible desde Internet en: www.iucnredlist.org (con acceso 13/01/10). 
18. JOHNSON, R.P. 1973. Scent marking in mammals. Animal Behaviour. 21:521-535.

19. KOMERS, P.E.; BROTHERTON, P.N. 1997. Dung pellets used to identify the distribution and density of the dik-dik. Afr. J. Ecol.. 35:124-132.

20. KREBS, C. 1999. Ecological methodology. Second Edition. University of British Columbia, Addison Wesley Longman Inc., 581p.

21. KREBS, C.H.J.; BOONSTRA, R.; NAMS, V.; O’DONOGHE, M.; HODGES, K.; BOUTIN, S. 2001. Estimating snowshoe hare population density from pellet plots: a further evaluation. Can. J. Zool. 79:1-4.

22. LANGBEIN, J.; HUTCHINGS, M.R.; HARRIS, S.; STOATE, C.; TAPPER, S.C.; WRAY, S. 1999. Techniques for assessing the abundance of brown hares Lepus europaeus. Mammal Rev. 29(2):93-116.

23. LINDENMAYER, D.B.; POSSINGHAM, H.P.; LACY, R.C.; MCCARTHY, M.A.; POPE, M.L. 2003. How accurate are population models? Lessons from landscape tests in a fragmented system. Ecol. Letters. 6:41-47.

24. LOMBARDI, L.; FERNÁNDEZ, N.; MORENO, S.; VILLAFUERTE, R. 2003. Habitat-related differences in rabbit (Oryctolagus cuniculus) abundance, distribution and activity. J. Mammalogy. 84(1):26-36.

25. LÓPEZ, L.; LÓPEZ, I.; MORA, J.; OSBAHR, K. 2000. Estudio preliminar del comportamiento de Dinomys branickii (Peters, 1873) en cautiverio. Rev. U.D.C.A Act. E Div. Cient. 3(1):28-35.

26. MCCARTHY, M.A.; ANDELMAN, S.; POSSINGHAM, H.P. 2003. Reliability of relative predictions in population viability analysis. Conserv. Biol. 17(4):982989.

27. MURRAY, D.L.; ROTH, J.D.; ELLSWORTH, E.; WIRSING, A.J.; STEURY, T.D. 2002. Estimating low-density snowshoe hare populations using fecal pellet counts. Can. J. Zool. 80(4):771-781.

28. MURRAY, D.L.; ELLSWORTH, E.; ZACK, A. 2005. Assessment of potential bias with snowshoe hare fecal pellet-plot. J. Wildlife Managem. 69(1):385395.

29. MYKYTOWYCZ, R.; GAMBALE, S. 1969. The distribution of dunghills and the behaviour of free living rabbits, Orytolagus cuniculus (L.), on them. Form and Function. 1:333-349.

30. NEFF, D.J. 1968. The pellet-group counting technique for big game trend, census and distribution: A review. J. Wildlife Managem. 32(3):597-614.

31. OSBAHR, K.; BAUTISTA, J.L. 1998. Contribución al conocimiento de un parásito nemátodo (Oxiuridae) de la guagua loba (Dinomys branickii). Rev. U.D.C.A Act. E Div. Cient. 1(1):34-42.

32. OSBAHR, K. 1999. Identificación de plantas consumidas por Agouti taczanowskii y Dinomys branickii a partir de fragmentos vegetales recuperados en heces. Rev. U.D.C.A Act. \& Div. Cient. 2:42-49.

33. OSBAHR, K.; RESTREPO, D. 2002. Determinación de calcio, hierro, proteína y otros requerimientos de nutrientes de Dinomys branickii (Peters 1873). Rev. U.D.C.A Act. \& Div. Cient. 4 (1):44-55.

34. OSBAHR, K.; ORTÍZ, D.; PÉREZ-TORRES, J. 2007. Amplitud de nicho y selectividad alimentaria del borugo de páramo (Cuniculus taczanowskii) (Stolzmann 1885) en un bosque andino nublado (Zipacón - Cundinamarca). Rev. U.D.C.A Act. E Div. Cient. 10(2):105-114.

35. OSBAHR, K.; ACEVEDO, P.; VILLAMIZAR, A.; ESPINOSA, D. 2009. Comparación de la estructura y de la función de los miembros anterior y posterior de Cuniculus taczanowskii y Dinomys branickii. Rev. U.D.C.A Act. \& Div. Cient. 12(1):37-51.

36. PALOMARES, F. 1993. Faecal marking behaviour by free-ranging common genets Genetta genetta and Egyptian mongooses Herpestes ichneumon in southwestern Spain. Zeitschrift für Säugetierkunde. 58:225-231.

37. PÉREZ-MEJÍA, S.; MANDUJANO, S.; MARTÍNEZ-ROMERO, L.E. 2004. Tasa de defecación del venado 
cola blanca, Odocoileus virginianus mexicanus, en cautividad en Puebla, México. Acta Zool. Mex. (n.s.). 20(3):167-170.

38. PUTMAN, R. J. 1984. Facts from feces. Mammal Rev. 14:79-97.

39. RINEY, T. 1957. The use of faeces counts in studies of several free-ranging mammals in New Zealand. New Zealand J. Sci. Techn. B. 38(6):507-532.

40. ROPER, T.J.; CONRADO, L.; BUTLER, J.; CHRISTIAN, S.E.; OSTLER, J.; SCHMID, T.K. 1993. Territorial marking with faeces in badgers Meles meles a comparison of boundary and hinterland latrine use. Behaviour. 127:289-307.

41. SCOTT MILLS, L.; GRIFFIN, P.C.; HODGES, K.E.; MCKELVEY, K.; RUGGIERO, L.; ULIZIO, T. 2005. Pellet count indices compared to mark-recapture estimates for evaluating snowshoe hare density. J. Wildlife Managem. 69(3):1053-1062.

42. SIMONETTI, J. 1989. Tasas de defecación y descomposición de fecas de Oryctolagus cuniculus en Chile Central. Medio ambiente 10(1):92-95.

43. SNEDDON, I.A. 1991. Latrine use in the European rabbit (Oryctolagus cuniculus). J. Mammalogy. 72:769-745.

44. SOKAL, R.R.; ROHLF, F.J. 2000. Biometry. Principles and Practices of Statistics in Biological Research. Tercera Edición. Freeman and Company. New York (USA). 887p.

45. SPRENT, J.A.; ANDERSEN, N.A.; NICOL, S.C. 2006. Latrine use by the short-beaked echidna (Tachyglossus aculeatus). Australian Mammalogy. 28:131-133.

46. STILLMAN, R.A.; CALDOW, R.W.G.; GOSS-CUSTARD, J.D.; ALEXANDER, M.J. 2000. Individual variation in intake rate: the relative importance of foraging efficiency and dominance. J. Anim. Ecol. 69:484-493.

47. SULKAVA, R.; MÄKELÄ, A.; KOTIAHO, J.S.; MÖNKKÖNEN, M. 2008. Difficulty of getting accurate and precise estimates of population size: the case of the Siberian flying squirrel in Finland. Ann. Zool. Fennici. 45:521-526.

48. THEUERKAUF, J.; ELLENBERG, H. 2000. Movements and defaecation of forest elephants in the moist semi-deciduous Bossematié Forest Reserve, Ivory Coast. Afr. J. Ecol. 38:258-261.

49. TUYTTENS, F.A.M.; LONG, B.; FAWCETT, T.; SKINNER, A.; BROWN, J.A.; CHEESEMAN, C.L.; RODDAM, A.W.; MACDONALD, D.W. 2001. Estimating group size and population density of Eurasian badgers Meles meles by quantifying latrine use. J. Appl.Ecol. 38:1114-1121.

50. URY, H.K. 1976. A comparison of four procedures for multiple comparisons among means (pairwise contrasts) for arbitrary sample sizes. Technometrics. 18:89-97.

51. VERNES, K. 1999. Pellet counts to estimate density of a rainforest kangaroo. Wildlife Soc. Bull. 27(4):991-996.

52. VUCETICH, J.A.; WAITE, T.A.; QVARNEMARK, L.; IBARGÜEN, S. 2000. Population variability and extinction risk. Conserv. Biol. 14:1704-1714.

53. WHITE, T.G.; ALBERICO, M.S. 1992. Dinomys branickii. Mammalian Species No. 410. The American Society of Mammalogists. p.1-5.

54. WOODS, C.A. 1984. Hystricognath rodents. En: Anderson, S.; Jones, K. Orders and families of recent mammals of the world. John Wiley and Sons, New York.,U.S.A. p.389-446.

55. ZAHRATKA, J.L.; BUSKIRK, S.W. 2007. Is size of fecal pellets a reliable indicator of species of leporids in the southern rocky mountains? J. Wildlife Managem. 71(6):2081-2083.

56. ZOLLNER, P.A.; SMITH, W.P.; BRENNAN, L.A. 1996. Characteristics and adaptive significance of latrines of swamp rabbits (Sylvilagus aquaticus). J. Mammalogy. 77(4):1049-1058.

Recibido: Febrero 3 de 2010

Aceptado: Abril 7 de 2010 